\title{
A Comprehensive Evaluation of Feature Selection for Gait Recognition Using Smartwatches
}

\author{
Neamah Al-Naffakh ${ }^{1,2}$, Nathan Clarke ${ }^{1,3}$, Paul Haskell-Dowland ${ }^{1,4}$, Fudong Li $^{1}$ \\ ${ }^{1}$ Centre for Security, Communications and Network Research, Plymouth University, Plymouth \\ ${ }^{2}$ Computer Science and Mathematics College, Kufa University \\ ${ }^{3}$ Security Research Institute, Edith Cowan University \\ ${ }^{4}$ School of Science, Edith Cowan University \\ United Kingdom ${ }^{1}$, Najaf, Iraq ${ }^{2}$, Perth, Western Australia ${ }^{3,4}$
}

\begin{abstract}
Activity recognition that recognises who a user is by what they are doing at a specific point of time is attracting an enormous amount of attention. Whilst previous research in activity recognition has focused on wearable dedicated sensors (body worn sensors) or using a smartphone's sensors (e.g. accelerometer and gyroscope), little attention is given to the use of wearable devices - which tend to be sensor-rich highly personal technologies. This paper presents a thorough analysis of the current state of the art in transparent and continuous authentication using acceleration and gyroscope sensors and an advanced feature selection approach to select the optimal features for each user. Two experiments are conducted; the first experiment used all the extracted features (i.e., 143 unique features) while (for comparison) a more selective set of only 30 features are used in the second experiment. The best results of the first experiment are average Euclidean distance scores of 0.55 and 1.41 for users' intra acceleration and gyroscope signals respectively and 3.33 and 5.85 for users' inter acceleration and gyroscope activities accordingly- providing sufficient disparity in distance to suggest a strong classification performance. In comparison, the second experiment demonstrated stronger results when evaluated (at best the average Euclidean distance scores is 0.03 and 0.19 for users' intra acceleration and gyroscope signals respectively and 1.65 and 1.1 for users' inter acceleration and gyroscope activities). The findings demonstrate that the technology is sufficiently capable and the nature of the signals captured sufficiently discriminative to be useful in performing activity recognition. Moreover, the proposed feature selection approach could offer better results and reduce the computational overhead on digital devices.
\end{abstract}

\section{Introduction}

Over 9.5 billion mobile devices, including smartphones and tablets, are currently utilized for various purposes (e.g., personal communication, and online payment). These devices are increasingly use to access sensitive information such as financial or health records [1]. The data that is stored in the mobile device is often considered more valuable than the cost of the device itself [2]. Therefore, securing information on these devices from unauthorized access in an effective and usable fashion is essential. However, current user authentication approaches (such as password and PIN) are considered as intrusive methods that hinder their usability and subsequently the security of the mobile device and its data [3]. According to a survey, $72 \%$ of their participants disabled the PIN code on their smartphones [4]; thus critical information that is stored on the device could be misused if it is lost or stolen. The use of a Transparent Authentication System is proposed in order to remove the user inconvenience (as the user is mainly transparently authenticated) and to improve the overall security in a continuous fashion [5]. Nevertheless, one of the key challenges for using transparent authentication is the lack of appropriate biometric modalities. In addition, previous research in this domain has also encountered performance issue due to the reliability of behavioural biometrics (i.e., the performance can be influenced by external environmental factors (e.g., mood)) [6].

Smartwatches have become more prevalent in the market and it is predicted that this trend will continue as the technology improves. A survey showed that more than $80 \%$ of smartwatch consumers said that healthy living and medical care access are major benefits of wearable technology [7]. Due to their fixed contact with individuals (i.e., either on left or right wrist), it is envisaged that smartwatches have the ability to capture more accurate personal data (e.g., acceleration and heart rate) than smartphones do. Therefore, wearables could be used to enhance mobile security in a more effective way. Most modern smartwatches contain Micro Electro Mechanical System sensors, which are based upon a single chip that offers both tri-axial gyroscope and accelerometer capabilities. Normally, gyroscopes (offering rotational velocities) and accelerometers (measuring non-gravitational accelerations) are used on their own for a biometric system. It is envisaged that the system performance can be improved if both of them are used together.

To this end, this paper explores the use of 
wearable computing devices for transparent authentication and in particular aims to investigate the feasibility of a novel Activity Recognition biometric modality. The rest of the paper is structured as follows: Section 2 reviews the state of the art in transparent and continuous authentication that uses acceleration and gyroscope sensors. Sections 3 and 4 present the data collection, feature extraction, preliminary results and the proposed feature selection approach. Sections 5 and 6 present the proposed architecture for an activity recognition system, the conclusions and future research directions.

\section{Background Literature}

Given the nature of wearable computing and its associated sensors, gait recognition is the modality that has the closest link to smartwatch-based activity recognition. Based upon how information is collected, gait recognition can be categorized into three main approaches: machine vision, wearable sensor, and mobile sensor. For the machine vision based approach, the movement of the human body is captured by using a fixed video-camera from a distance (such as CCTV) and it is mainly used for the purpose of identification.

Table 1. Comprehensive Analysis on Gait Authentication using Wearable and Mobile Sensors (C: Cycle-based; S: Segment-based; SF: Statistical Features; CF: Coefficient Features; DTW: Dynamic Time Warping; HMM: Hidden Markov Model; SVM: Support Vector Machine; EER: Equal Error

Rate; CCR: Correct Classification Rate; SD, CD Same and Cross Day)

\begin{tabular}{|l|l|l|c|c|c|c|}
\hline & & & & & \\
& & & & & \\
\end{tabular}

In comparison, the other two approaches focus upon capturing the periodic motion of the legs by attaching physical recording sensors on the human body such as hip, waist, lower leg, and arm or by carrying a mobile on the go; they are mainly used to verify the identity of the carrier. It is these studies that this review will focus upon. A comprehensive analysis of the prior studies on gait authentication using wearable and mobile sensors is summarized in Table 1. The use of wearable sensors that are used to collect gait signals created a new domain for transparent and continuous user authentication on mobile devices. However, these studies are required to use specialized devices that are expensive for collecting the gait information; and the volume of their data per user is somewhat limited (i.e., 60 to 300 seconds) as illustrated in Table 1. Moreover, due to the complexity of the data collecting device, an additional cost would be required if they were utilised in a real-world system. Therefore, more recent studies attempted to utilize the smartphone built-in sensors for gathering the gait signal; as no extra cost is required. Also, this permits the authentication task to be performed in a transparent and continuous manner as the smartphone is carried in the user's pocket [11-17].

A large body of research on accelerometer-based activity recognition by using the Same-day scenario (i.e., the training and testing data is collected on the same day) exist. In comparison, little work is considered by applying the Cross-day evaluation scenario (which is a more realistic test as it shows the variability of the human gait behaviour over the time). Most research claim a system resilient to the cross-day problem either trains on data from trials that are also used to test (thus not making it a true cross-day system) or has a high error rate, preventing the system being used practically. The lack of realistic data underpins a significant barrier in applying gait recognition in practice.

To extract gait features from the captured signal, previous studies have focused upon two main approaches: cycle-based and segment-based. The former attempts to detect the periodic steps of the individuals by standardizing the number of steps as opposed to the amount of time represented in each instance (i.e., pace independent). The latter focuses on fixed-length blocks of data (without prior identification of the contained gait cycles). The literature shows that the performance varies significantly by using these two methods. The cycle extraction purportedly offers an exciting opportunity if a system is implemented effectively and trained in just a manner of steps; however, the error rate of using this approach is considered as high: the EER is ranging from $20.1 \%$ [11] to $33.3 \%$ [16] as demonstrated in Table 1. The high error rate is most likely caused by the result of the complicated and unclear nature of cycle extraction, as gait is only semi-periodic and the signals originating from smartphones are noisy due to a number of factors (e.g., the device not being securely fastened to the 
user, cheap sensors, and rounding errors). Furthermore, cycles are not guaranteed to be the same length and can vary widely in length depending on the pace of how a user walks; cycle extraction must be paired with a system that normalizes the length of each step, which adds another parameter to be configured and constantly refined. In contrast, the segmentation based method focuses on fixed-length blocks of gait data. While the segmentation based method is simple to implement, there is no guarantee on how many steps are completed within a given time window (there could be no full step at all). However, the performance of the segment based method appears to be more effective and stable, with studies reporting EERs between $6.1 \%$ and $10 \%$ [13, 15]. If the CCR were used, the performance of segment based method is even better: in the range of $93 \%-100 \%$ of the CCR $[12,18,19]$.

With respect to features, several studies have suggested that both statistical features (e.g., standard deviation, average, and N-bin histogram) and cepstral coefficient features (e.g., Mel Frequency Cepstral Coefficients (MFCCs) and Bark Frequency Cepstral Coefficients (BFCCs)) can be used to produce better performance [12, 13, 15, 18, 19, 20]. In addition, some studies only used the combination of MFCCs and BFCCs features alone and still managed to produce a good level of results $[13,15]$. The improvement on the performance of sensor based biometric systems can be attributed to more intricate feature vectors that utilize more complex features (e.g. MFCC and BFCC).

In terms of matching/classification, several classification methods (e.g., Absolute, Euclidean, and Neural Networks) can be used for training and testing phases. Many researchers prefer traditional approaches where a single template is generated and is later tested based upon the similarity between the template and the test data. By using this principle, various EERs between $5.7 \%$ and $33.3 \%$ were obtained from the following studies $[8,9,10,11,14$, 16 , 17]. While this approach works well for physiological biometric methods (e.g., face or fingerprint), it is less effective for behavioural biometric techniques (e.g., body movement and keystroke dynamics). This is because the user's behaviour can change over time and be affected by other factors (e.g., mood and health). Therefore, it is more reasonable to collect user's multiple instances on multiple days and apply more complex algorithms (e.g., HMM and Neural Networks) upon them for generating the template and performing the classification process. Recent studies on mobile accelerometer-based gait authentication and smartwatch-based activity recognition demonstrate that promising results are obtained by using advanced techniques (e.g., decision-tree based classifiers, and neural networks) $[12,13,15,17,18$, 19].
Based upon the classification result, a decision on whether to accept or reject the output is made by the system. Accordingly to the literature, two standard schemas are used: majority or quorum voting. A better performance is normally obtained by using the quorum voting technical while the system is more resilient to error when the majority voting is applied. Under the quorum voting scheme, a small number of correct classification outputs are required to accept a user. While this will improve the user convenience (i.e., the user will be highly likely to accept the deployment of such system), it will result in a high false acceptance rate (i.e., there is a high chance for the imposter to abuse the system). In contrast, more discriminative user behaviour is required when utilizing the majority voting technique; otherwise, a high false rejection rate will be produced by the system. It is understood that the system will provide better security when using the majority voting method; at the same time, the system is more intrusive (i.e., less user friendly). As a result, it is important that a proper decision logic that can balance the system security and user convenience is applied for the gait authentication system.

The majority of previous studies collected the user's movement data by placing a smartphone in a fixed position (e.g., in the trouser pocket or on the hip). It is widely understood that smartphones suffer from several issues to produce a consistent and reliable data collection in real life; these include the problem of orientations (i.e., screen rotations) and off-body carry (e.g., when the device is carried in a handbag), making the data collection process less accurate or nearly impossible. In contrast, smartwatches provide a more consistent user's motion data collection as it is almost fixed to the user (i.e., it is worn on either left or right hand) regardless of their clothing choices. In addition, the smartwatch can provide a consistent orientation (i.e., it is worn in such a way that the text on screen is easily readable to the user). As a result, smartwatches offer the opportunity to collect the user's motion data in a more effective and reliable fashion than smartphones could.

\section{Preliminary Analysis of Activity Recognition}

With the aim of investigating the feasibility of using wearable computing for transparent user authentication, a preliminary study was conducted to capture and analyse the user's movement data. Details of the study, including data collection, feature extraction and analysis are presented in the following subsections. 


\subsection{Data Collection and Transformation}

In order to collect user's movement data, the Microsoft band 2 was utilized due to its wide range of built-in sensors. Of specific interest in this study were the accelerometer and gyroscope sensors, where samples were collected at a rate of 30-32 samples per second for the $\mathrm{x}, \mathrm{y}$ and $\mathrm{z}$-axes. As soon as the data was collected by the smartwatch, it was sent to a smartphone residing in the user's pocket via Bluetooth. In total, 36 users participated for the data collection; each user was required to walk on a predefined route over six sessions, each of the three sessions were provided on different days within a time frame of 3 weeks. In each session, the subject was asked to walk at a natural speed on flat ground for 2 minutes. For a more realistic scenario, the subject had to stop in order to open a door, and take multiple turns. Once the data collection was completed, the signal processing phase was undertaken- a brief description of the steps are:

- Time interpolation: Due to the limited accuracy of sensors in android devices, the smartwatch was not able to record data at a fixed sample rate (in other words, the time intervals between two successive acceleration values were not fixed). Therefore, time interpolation was required to make sure that the time period between two successive data points was always equal.

- Filtering: a low pass filter was designed in order to enhance the accuracy of the signal. This was done by setting the cut-off frequency to $0.2 \mathrm{~Hz}$.

- Segmentation: once the signal was filtered, the tri-axial raw format for both acceleration and gyroscope signals were segmented into 10second segments by using a sliding window approach with no overlapping. Therefore, in total 36 samples were collected for each user per day.

\subsection{Feature extraction}

In the previous work [20], 88 features were extracted for the gait data based upon prior work identified in gait recognition studies $[12,13,15,17,18,19]$. In this study, a comprehensive feature extraction process was carried out on both the acceleration and gyroscope data. Features were extracted in both, the time and frequency domains.

In total, 143 unique features were created for each sensor. Details of these features (e.g., what they are and how they are calculated) are presented below; also the number of generated features for each type is specified in brackets.

\subsubsection{Time domain features}

- Difference (3): the difference between the maximum and minimum of the values in the segment (each axis).

- Median (3): the median values of the data points in the segment.

- Zero crossing rate (3): is the rate of signchanges along a signal.

- Root Mean Square (3): the square root of the mean squared.

- Interquartile range (3): is the range in the middle of the data. It is the difference between the upper and lower quartiles in the segment.

- Skewness (3): is a measure of the symmetry of distributions around the mean value of the segment.

- Kurtosis (3): is a measure of the shape of the curve for the segment data.

- Percentile25 (3): the percentile rank is measured using the formula: $\mathrm{R}=(\mathrm{P} / 100)^{*}(\mathrm{~N}+1)$. Where $\mathbf{R}$ represents rank order of values, $\mathbf{P}$ percentile rank, $\mathbf{N}$ total number of the data points in the segment.

- Percentile50 (3) : similar to the previous feature but setting $\mathrm{P}=50$

- Maximum (3): The largest 4 values in the segment are calculated and averaged.

- Minimum (3): The smallest 4 values in the segment are calculated and averaged.

- Correlation Coefficients (3): The relationship between two axes is calculated. The Correlation Coefficients is measured between $\mathrm{X}$ and $\mathrm{Y}$ axes, $\mathrm{X}$ and $\mathrm{Z}$ axes, and $\mathrm{Y}$ and $\mathrm{Z}$ axes.

- Average (3): the mean of the values in the segment.

- Standard Deviation (3): the Standard Deviation of the values in the segment.

- Average Absolute Difference (3): the average absolute distance of all values in the segment from the mean value over the number of data point in the segment.

- Time Between Peaks (3): during the user's walking, repetitive peaks are generated in the signal. Thus, the time between consecutive peaks was calculated and averaged.

- Minimum Peaks (3): the smallest 4 peaks in the segment are calculated and averaged.

- Maximum Peaks (3): the largest 4 peaks in the segment are measured and averaged.

- Peaks Occurrence (3): determines how many peaks are in the segment.

- Binned Distribution (30): relative histogram distribution in linear spaced bins between the minimum and the maximum acceleration in the segment. Ten bins are used for each axis. 
- Average Resultant Acceleration (1): for each value in the segment of $\mathrm{x}, \mathrm{y}$, and $\mathrm{z}$ axes, the square roots of the sum of the values of each axis squared over the segment size (i.e., 10 seconds) are calculated.

- Variance (3): The second-order moment of the data.

3.2.2 Frequency domain features. The process of extracting frequency domain features is somewhat different from the time domain. Before extracting a frequency domain feature, a Fourier transform needs to be applied to the data. A set of frequency domain features are calculated which might be useful to create a discriminative feature vector for each individual. The extracted features are presented in Table 2. In the second and the fourth columns, NF stands for the number of generated features.

Table 2. Frequency domain features

\begin{tabular}{|c|c|c|l|}
\hline Features & NF & Features & NF \\
\hline Energy & $\mathbf{3}$ & Difference & $\mathbf{3}$ \\
\hline Entropy & $\mathbf{3}$ & Zero crossing rate & $\mathbf{3}$ \\
\hline Root Mean Square & $\mathbf{3}$ & Interquartile range & $\mathbf{3}$ \\
\hline Maximum & $\mathbf{3}$ & Correlation Coefficients & $\mathbf{3}$ \\
\hline Minimum & $\mathbf{3}$ & Percentiles25 & $\mathbf{3}$ \\
\hline Standard Deviation & $\mathbf{3}$ & Percentiles 50 & $\mathbf{3}$ \\
\hline Median & $\mathbf{3}$ & Skewness & $\mathbf{3}$ \\
\hline Variance & $\mathbf{3}$ & Kurtosis & $\mathbf{3}$ \\
\hline $\begin{array}{c}\text { Average Absolute } \\
\text { Difference }\end{array}$ & $\mathbf{3}$ & $\begin{array}{c}\text { Average Resultant } \\
\text { Acceleration }\end{array}$ & $\mathbf{1}$ \\
\hline
\end{tabular}

3.2.3 Validating features extracted from the smartwatch. In order to validate the effectiveness of the 143 generated features for a promising authentication technique, the data set was divided to form both reference and testing templates for all users in two scenarios (i.e., Same-Day and CrossDay). The average Euclidean distance between the reference template and testing templates was calculated; this distance value represents the similarity between the two templates: the smaller the value, the more similarity between the reference and testing templates and vice versa. As a result, in order for this technique to work, a small distance value should be presented when the reference and testing templates are from the same user; while a large distance value should be expected when these templates are from different users - representing the intra and inter sample variances. The results of 36 users' movement data for the Same-Day and CrossDay scenarios are presented in Tables 3 and 4 respectively.

Table 3 shows (for the Same-Day) the acceleration templates of the same user competitive average Euclidean distance scores, ranging from 0.55 (subject 17) to 1.41 (subject 34). When gyroscope data was used, the distance scores of the same subject were in the range of 1.41 (subject 35) - 4.61 (subject 14). In comparison, average Euclidean distance scores for reference and testing templates of different subjects that are extracted on the same day are much larger: 2.54 (subject 25) to 3.33 (subject 34) for acceleration and 3.57 (subject 4) - 5.85 (subject 20) for gyroscope.

The imposter distance scores from each genuine user are further analyzed separately (Figures 1 and 2 for acceleration and gyroscope data respectively). The given acceleration based- results in Figure 1 show that the user's arm movement is highly consistent and each subject has a distinctive arm pattern. Moreover, the majority of imposters are more likely to be rejected by the system as their distances scores were far away from the genuine user. In contrast, when gyroscope data was applied, the average Euclidian distance scores between imposters and a genuine user were greatly dependent on the subject (Figure 2). For example, subjects 3, 5, $7,11,13,15,17,21,27$, and 33 had low intervariance, which means the chance of accepting an imposter is high. One reason for this is that using a large number of features might influence the system performance.

Table 3. Results of Same-Day Scenario

\begin{tabular}{|c|l|l|c|c|c|c|c|c|c|c|}
\hline \multirow{2}{*}{ ID } & \multicolumn{2}{|l|}{ Dist to Self } & \multicolumn{2}{|c|}{ Dist to Others } & \multirow{2}{*}{ ID } & \multicolumn{2}{l|}{ Dist to Self } & \multicolumn{2}{c|}{ Dist to Others } \\
\cline { 2 - 7 } & Acc & Gyr & Acc & Gyr & & Acc & Gyr & Acc & Gyr \\
\hline $\mathbf{1}$ & 0.89 & 2.08 & 2.76 & 3.82 & $\mathbf{1 9}$ & 0.97 & 1.96 & 3.08 & 4.45 \\
\hline $\mathbf{2}$ & 0.72 & 1.82 & 3.3 & 3.72 & $\mathbf{2 0}$ & 1.58 & 2.2 & 3.13 & 5.85 \\
\hline $\mathbf{3}$ & 0.77 & 3.21 & 3.18 & 4.03 & $\mathbf{2 1}$ & 0.83 & 2.45 & 2.62 & 3.51 \\
\hline $\mathbf{4}$ & 1.11 & 2.2 & 3.07 & 3.57 & $\mathbf{2 2}$ & 0.74 & 1.53 & 2.82 & 3.74 \\
\hline $\mathbf{5}$ & 0.89 & 2.35 & 2.69 & 3.75 & $\mathbf{2 3}$ & 1.32 & 2.96 & 2.68 & 5.13 \\
\hline $\mathbf{6}$ & 1.01 & 1.73 & 2.65 & 3.84 & $\mathbf{2 4}$ & 1.04 & 2.35 & 2.72 & 3.73 \\
\hline $\mathbf{7}$ & 1.15 & 2.57 & 2.78 & 3.7 & $\mathbf{2 5}$ & 1.01 & 1.76 & 2.54 & 3.74 \\
\hline $\mathbf{8}$ & 1.02 & 2.56 & 2.67 & 4.14 & $\mathbf{2 6}$ & 0.91 & 1.91 & 3.13 & 3.72 \\
\hline $\mathbf{9}$ & 0.84 & 1.78 & 2.7 & 3.61 & $\mathbf{2 7}$ & 1.17 & 3.05 & 3.69 & 3.97 \\
\hline $\mathbf{1 0}$ & 1.18 & 2.21 & 2.84 & 3.9 & $\mathbf{2 8}$ & 1.12 & 2.23 & 2.58 & 3.61 \\
\hline $\mathbf{1 1}$ & 1.19 & 4.94 & 2.93 & 5 & $\mathbf{2 9}$ & 1.2 & 2.18 & 2.89 & 3.8 \\
\hline $\mathbf{1 2}$ & 0.76 & 2.35 & 2.57 & 4 & $\mathbf{3 0}$ & 1.02 & 2 & 2.8 & 3.59 \\
\hline $\mathbf{1 3}$ & 1.02 & 3.9 & 2.71 & 4.82 & $\mathbf{3 1}$ & 1.01 & 1.9 & 2.85 & 3.72 \\
\hline $\mathbf{1 4}$ & 1.23 & 4.61 & 3.17 & 5.24 & $\mathbf{3 2}$ & 0.89 & 2.33 & 3.06 & 3.49 \\
\hline $\mathbf{1 5}$ & 0.98 & 2.44 & 2.83 & 3.72 & $\mathbf{3 3}$ & 0.86 & 2.95 & 2.73 & 3.79 \\
\hline $\mathbf{1 6}$ & 1.4 & 1.87 & 3.23 & 4.78 & $\mathbf{3 4}$ & 1.41 & 3.14 & 3.33 & 4.64 \\
\hline $\mathbf{1 7}$ & 0.55 & 3.59 & 2.91 & 4.5 & $\mathbf{3 5}$ & 0.91 & 1.41 & 2.62 & 3.93 \\
\hline $\mathbf{1 8}$ & 0.97 & 2.39 & 2.92 & 4.72 & $\mathbf{3 6}$ & 0.85 & 1.51 & 2.57 & 4.07 \\
\hline
\end{tabular}

A more realistic test for a behavioural basedbiometric comes when the Cross-day scenario is applied to show the influence of the variation of human movement over time. Therefore, the Crossday scenario was also evaluated and the results shown in Table 4. 


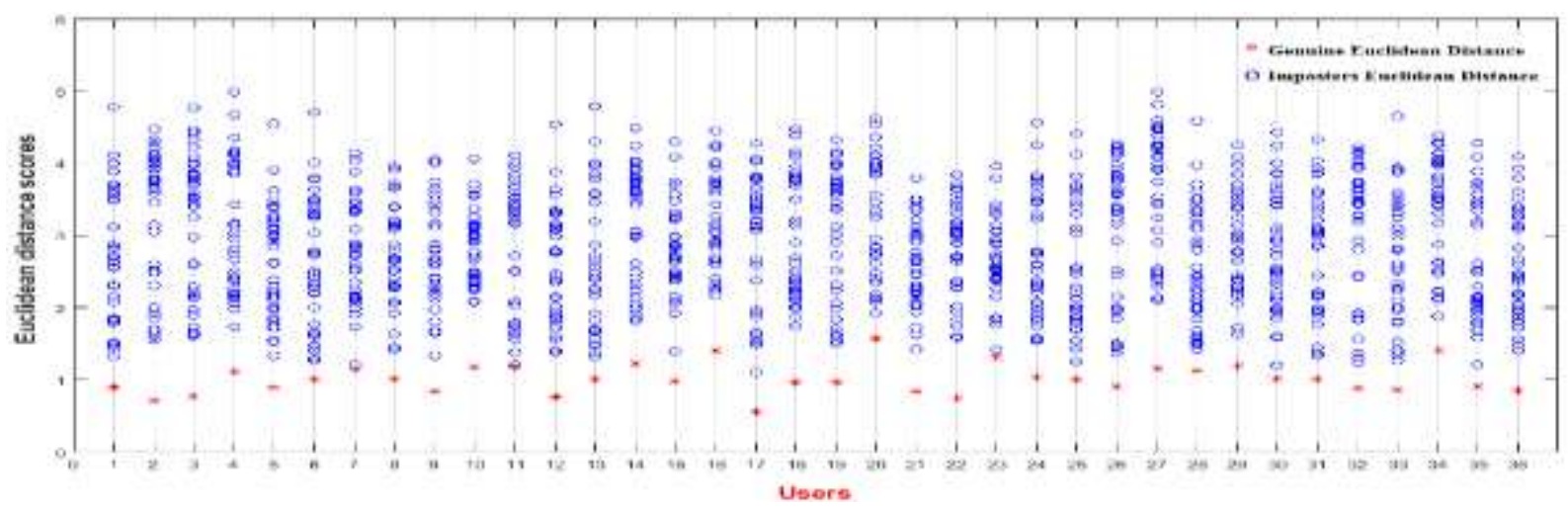

Figure 1. Acceleration Euclidean Distance Scores Using All Features

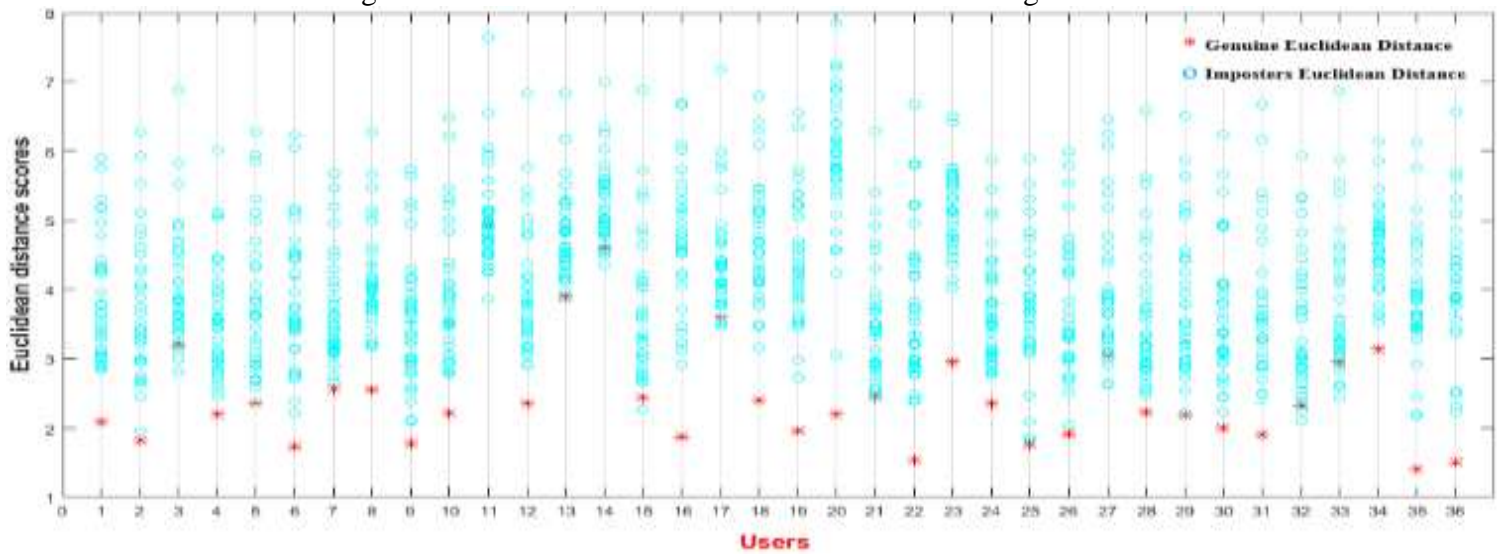

Figure 2. Gyroscope Euclidean Distance Scores Using All Features

While the distance scores under this more realistic evaluation scenario for acceleration and gyroscope templates of the genuine user were increased, they were still viable to be used for discriminating users: ranging from 0.58 (subject 17) to 2.11 (subject 10) for acceleration and from 1.52 (subject 22) to 4.51 (subject 13) for gyroscope. In comparison, the resulting distance scores for reference and probe templates of imposters were generally quite high: 2.47 (subject 25) to 3.39 (subject 27) for acceleration, which is an indication that imposters are more likely to be rejected by the system. In contrast, the distance scores for the gyroscope were slightly larger ranging from 3.6 (subject 9) to 6.21(subject 20); this could cause more imposters to be falsely accepted. The results also show the necessity of using a sensor fusion approach (i.e., combining the smartwatch sensors data) in order to have a balance between security and usability. In addition, an improved feature selection method to select a set of features that have low-intra and high inter-variance is definitely required.

Table 4. Results of Cross-Day Scenario

\begin{tabular}{|c|c|c|c|c|c|c|c|c|c|}
\hline \multirow{2}{*}{ ID } & \multicolumn{2}{|c|}{ Dist to Self } & \multicolumn{2}{|c|}{ Dist to Others } & \multirow{2}{*}{ Dist to Self } & \multicolumn{2}{|c|}{ Dist to Others } \\
\cline { 2 - 7 } \cline { 6 - 9 } & Acc & Gyr & Acc & Gyr & & Acc & Gyr & Acc & Gyr \\
\hline $\mathbf{1}$ & 1 & 3.28 & 2.65 & 4.5 & $\mathbf{1 5}$ & 1.69 & 3.9 & 2.67 & 4.38 \\
\hline $\mathbf{2}$ & 0.91 & 1.94 & 3.29 & 3.83 & $\mathbf{1 6}$ & 1.33 & 2.1 & 2.93 & 4.49 \\
\hline
\end{tabular}

\begin{tabular}{|c|l|c|c|c|c|c|c|c|c|}
\hline $\mathbf{3}$ & 0.9 & 3.38 & 3.09 & 3.61 & $\mathbf{1 7}$ & 0.58 & 4.0 & 2.91 & 4.29 \\
\hline $\mathbf{4}$ & 1.16 & 2.43 & 2.94 & 3.68 & $\mathbf{1 8}$ & 1.12 & 2.3 & 2.76 & 4.18 \\
\hline $\mathbf{5}$ & 1.05 & 2.29 & 2.62 & 4.15 & $\mathbf{1 9}$ & 1.2 & 2.9 & 2.91 & 4.27 \\
\hline $\mathbf{6}$ & 0.95 & 1.58 & 2.63 & 3.85 & $\mathbf{2 0}$ & 1.4 & 2.7 & 2.68 & 6.21 \\
\hline $\mathbf{7}$ & 1.03 & 2.71 & 2.76 & 3.81 & $\mathbf{2 1}$ & 1.0 & 2.4 & 2.62 & 4.5 \\
\hline $\mathbf{8}$ & 0.97 & 3.19 & 2.67 & 4.3 & $\mathbf{2 2}$ & 1.0 & 1.5 & 2.63 & 3.83 \\
\hline $\mathbf{9}$ & 1.1 & 1.86 & 2.63 & 3.6 & $\mathbf{2 3}$ & 1.2 & 4.1 & 2.6 & 3.61 \\
\hline $\mathbf{1 0}$ & 2.11 & 3.1 & 3 & 4.18 & $\mathbf{2 4}$ & 0.9 & 3.3 & 2.74 & 3.68 \\
\hline $\mathbf{1 1}$ & 1.13 & 3.8 & 2.83 & 4.39 & $\mathbf{2 5}$ & 1.3 & 1.6 & 2.47 & 4.15 \\
\hline $\mathbf{1 2}$ & 0.89 & 2.81 & 2.59 & 4.1 & $\mathbf{2 6}$ & 1.0 & 2 & 3.12 & 3.85 \\
\hline $\mathbf{1 3}$ & 1.1 & 4.51 & 2.69 & 4.87 & $\mathbf{2 7}$ & 1.2 & 1.9 & 3.39 & 3.81 \\
\hline $\mathbf{1 4}$ & 0.85 & 4.18 & 3.14 & 5.69 & $\mathbf{2 8}$ & 1.0 & 3.9 & 2.52 & 4.3 \\
\hline $\mathbf{2 9}$ & 1.4 & 2.6 & 2.91 & 3.6 & $\mathbf{3 3}$ & 0.9 & 2.7 & 2.66 & 4.87 \\
\hline $\mathbf{3 0}$ & 0.8 & 2.1 & 2.68 & 4.18 & $\mathbf{3 4}$ & 1.1 & 2.7 & 3.32 & 5.69 \\
\hline $\mathbf{3 1}$ & 1.1 & 2.0 & 2.79 & 4.39 & $\mathbf{3 5}$ & 1.0 & 2.0 & 2.64 & 4.38 \\
\hline $\mathbf{3 2}$ & 0.8 & 2.1 & 3.04 & 4.1 & $\mathbf{3 6}$ & 0.8 & 1.5 & 2.55 & 4.49 \\
\hline
\end{tabular}

\section{Feature selection approach}

The feature selection step has become the focus of many research studies in the area of authentication in order to reduce potentially large dimensionality of input data and thus system performance could be enhanced by selecting the most optimal and unique features for individual. Furthermore, it will be easier to manipulate small feature subsets on digital devices (i.e., smartphones and smartwatches). The majority of activity recognition systems select common features (e.g., features that have the smallest standard deviation) for all the population. 
This could be very useful if it is considered that the authentication system is based on identifying the genuine user only. However, a balance between security and usability needs to be taken for Transparent Authentication Systems (i.e., low false acceptance rate (FAR) and low false rejection rate (FRR)). FAR shows the percentage in which the system incorrectly accepts an imposter as the legitimate user while FRR displays the percentage in which the authorized user is wrongly rejected by the system.

The current study focused on creating a dynamic feature vector that contains unique features for each subject. This was achieved by measuring the standard deviation (STD) for each feature and, subsequently, selecting feature subsets that have the smallest STD for each user independently. Using this method 30 features were identified for each subject. For example, the reference template of subject 1 could be created by using features $1,2,3$, and 7 (features with smallest STD) while features 3, 4, 5, and 7 might be used to form the reference template of subject 2. This could result in low FRR and FAR. Moreover, selecting small feature subsets will greatly reduce the complicated computations on smartphones, which limit processing resources as compared to standard computers.

To evaluate the effectiveness of the selected feature subsets (30 features) for classification, the Euclidean distance metric for both scenarios (i.e., Same-Day and Cross-Day) are calculated and the results were presented in Tables 5 and 6 accordingly. The results in Table 5 indicate that applying small feature subsets yields very small distance scores between the training and test of the genuine user ranging from 0.03 (subject 6) to 0.2 (subject 16) for acceleration and 0.19 (subject 22) to 0.39 (subject 18) for gyroscope (compared to 0.55 and 1.41 for acceleration and 1.41 to 3.59 for gyroscope when the entire feature sets are used). These results suggest that the chance of a genuine user being correctly authenticated by the system is high. Also, the system would be able to identify imposters as their Euclidean distance scores are large: 0.57 (subject 28 ) to 1.65 (subject 27) and 0.48 (subject 26) to 1.1 (subject 15) for acceleration and gyroscope respectively. Interestingly, the results in Table 6 show that the selected feature subsets are more resistant to changes of the user's behavior as the Euclidean distance scores of Same and Cross-day scenarios for most subjects are nearly similar, apart from subjects $10,15,25,27,29$, and 31 for acceleration and subjects 9, 10, 15, and 23 for gyroscope.

By using features associated with the acceleration data, Figure 3 shows that all imposters will be more likely to be rejected by the system (apart from subject 8 as one or two imposters might be able to deceived the system).
Table 5. Results of Same-Day Scenario by using 30 Features

\begin{tabular}{|c|c|c|c|c|c|c|c|c|c|}
\hline \multirow{2}{*}{ ID } & \multicolumn{2}{|c|}{ Dist to Self } & \multicolumn{2}{|c|}{ Dist to Others } & \multirow{2}{*}{ ID } & \multicolumn{2}{|c|}{ Dist to Self } & \multicolumn{2}{|c|}{ Dist to Others } \\
\hline & Acc & Gyr & Acc & Gyr & & \begin{tabular}{|l|} 
Acc \\
\end{tabular} & Gyr & Acc & Gyr \\
\hline 1 & 0.08 & 0.32 & 0.99 & 1.06 & 19 & 0.14 & 0.25 & 0.78 & 0.51 \\
\hline 2 & 0.06 & 0.23 & 1.05 & 0.5 & \begin{tabular}{|l|}
20 \\
\end{tabular} & \begin{tabular}{|l|l|}
0.11 \\
\end{tabular} & 0.36 & 1.02 & 1 \\
\hline 3 & 0.07 & 0.23 & 1.18 & 0.84 & 21 & 0.06 & 0.26 & 0.82 & 0.56 \\
\hline 4 & 0.08 & 0.29 & 0.91 & 0.56 & 22 & 0.04 & 0.19 & 0.84 & 0.95 \\
\hline 5 & 0.05 & 0.26 & 0.9 & 0.52 & 23 & 0.1 & 0.29 & 0.9 & 0.64 \\
\hline 6 & 0.03 & 0.25 & 0.7 & 1.03 & 24 & 0.08 & 0.3 & 0.71 & 0.51 \\
\hline 7 & 0.09 & 0.3 & 0.83 & 0.58 & 25 & 0.06 & 0.24 & 0.88 & 0.55 \\
\hline 8 & 0.1 & 0.3 & 0.69 & 0.53 & 26 & 0.06 & 0.3 & 0.89 & 0.48 \\
\hline 9 & 0.07 & 0.2 & 0.7 & 0.5 & 27 & \begin{tabular}{|l|l|} 
\\
\end{tabular} & \begin{tabular}{|l}
0.29 \\
\end{tabular} & 1.65 & 0.94 \\
\hline 10 & 0.15 & 0.2 & 1.02 & 0.95 & 28 & 0.07 & 0.21 & 0.57 & 0.8 \\
\hline 11 & 0.1 & 0.3 & 1.06 & 0.59 & 29 & 0.08 & 0.23 & 0.58 & 1.05 \\
\hline 12 & 0.04 & 0.21 & 0.97 & 0.89 & 30 & 0.08 & 0.25 & 0.76 & 0.91 \\
\hline 13 & 0.08 & 0.33 & 0.65 & 0.59 & 31 & 0.06 & 0.24 & 0.65 & 0.53 \\
\hline 14 & 0.08 & \begin{tabular}{|l}
0.27 \\
\end{tabular} & 1 & 0.62 & 32 & 0.05 & 0.21 & 1 & 0.84 \\
\hline 15 & 0.09 & 0.23 & 0.71 & 1.1 & 33 & 0.05 & 0.25 & 0.77 & 1.07 \\
\hline 16 & 0.2 & \begin{tabular}{|l}
0.33 \\
\end{tabular} & 1.16 & 0.68 & 34 & \begin{tabular}{|l|l|} 
\\
\end{tabular} & \begin{tabular}{|l|l} 
\\
\end{tabular} & 1.12 & 0.61 \\
\hline 17 & 0.05 & 0.31 & 0.9 & 0.56 & 35 & 0.1 & 0.24 & 0.85 & 0.58 \\
\hline 18 & 0.13 & 0.39 & 1.1 & 0.99 & 36 & 0.07 & 0.25 & 0.86 & 0.89 \\
\hline
\end{tabular}

Table 6. Results of Cross-Day Scenario by using 30 Features

\begin{tabular}{|c|c|c|c|c|c|c|c|c|c|}
\hline \multirow{2}{*}{ ID } & \multicolumn{2}{|c|}{ Dist to Self } & \multicolumn{2}{|c|}{ Dist to Others } & \multirow{2}{*}{ ID } & \multicolumn{2}{|c|}{ Dist to Self } & \multicolumn{2}{|c|}{ Dist to Others } \\
\hline & \begin{tabular}{|l|} 
Acc \\
\end{tabular} & Gyr & Acc & Gyr & & Acc & Gyr & Acc & Gyr \\
\hline 1 & 0.09 & 0.31 & 0.9 & 1.08 & 19 & 0.17 & 0.31 & 0.74 & 0.51 \\
\hline 2 & \begin{tabular}{|l|l|}
0.11 \\
\end{tabular} & 0.26 & 1.15 & 0.53 & 20 & 0.18 & 0.35 & 0.64 & 0.93 \\
\hline 3 & 0.12 & 0.25 & 1.19 & 0.55 & 21 & 0.06 & 0.27 & 0.81 & 0.62 \\
\hline 4 & \begin{tabular}{|l|l|}
0.1 \\
\end{tabular} & 0.28 & 0.69 & 0.59 & 22 & 0.05 & 0.22 & 0.95 & 1 \\
\hline 5 & 0.12 & 0.31 & 1 & 0.48 & 23 & 0.17 & 0.48 & 0.74 & 0.83 \\
\hline 6 & 0.05 & 0.23 & 0.69 & 0.99 & 24 & 0.11 & 0.38 & 0.88 & 0.57 \\
\hline 7 & 0.07 & 0.31 & 0.83 & 0.61 & 25 & 0.21 & 0.25 & 0.46 & 0.59 \\
\hline 8 & 0.09 & 0.31 & 0.75 & 0.5 & 26 & 0.12 & 0.29 & 0.56 & 0.51 \\
\hline 9 & 0.12 & 0.3 & 0.79 & 0.52 & 27 & 0.2 & 0.26 & 0.67 & 1.19 \\
\hline 10 & 0.28 & 0.31 & 1.1 & 0.99 & 28 & 0.08 & 0.23 & 0.54 & 0.97 \\
\hline 11 & 0.13 & 0.29 & 1.02 & 0.61 & 29 & 0.17 & 0.27 & 0.95 & 1.07 \\
\hline 12 & 0.05 & 0.22 & 0.99 & 1.01 & 30 & \begin{tabular}{|l|l|} 
& 0.1 \\
\end{tabular} & 0.3 & 0.86 & 0.48 \\
\hline 13 & 0.08 & 0.31 & \begin{tabular}{|l|l}
0.67 \\
\end{tabular} & 0.66 & 31 & 0.12 & 0.23 & 0.5 & 0.57 \\
\hline 14 & 0.09 & 0.31 & 0.69 & 0.65 & 32 & 0.05 & 0.25 & 0.98 & 0.96 \\
\hline 15 & 0.27 & 0.39 & 0.78 & 1.15 & 33 & 0.1 & 0.3 & 0.77 & 1.03 \\
\hline 16 & 0.18 & 0.3 & 1.01 & 0.62 & 34 & 0.18 & 0.29 & 1.09 & 0.65 \\
\hline 17 & 0.06 & 0.27 & 0.88 & 0.59 & 35 & 0.12 & 0.27 & 0.86 & 0.86 \\
\hline 18 & 0.17 & \begin{tabular}{|l|}
0.3 \\
\end{tabular} & 1.04 & 0.77 & 36 & 0.08 & 0.26 & 0.85 & 0.8 \\
\hline
\end{tabular}

When gyroscope features are used, Figure 4 reveals that the system was still able to identify the majority of imposters. While some of the gyroscope results may not seems that positive, they are actually quite impressive when one considers that they were produced from only 30 features. Compared to the previous experiment, which used the whole gyroscope feature set (143 features), it can be clearly noticed that the imposters overlapping with subjects $3,5,11,15,21,27$, and 33 are greatly reduced. This is due to the fact that selecting more discriminative feature sets could result in low intra-variance and high inter-variance. The results show that accelerometer features are unique and more 
distinctive than gyroscope features as the distance scores between the reference and test templates of the genuine user are small (i.e., low intra- variance), as well as provide a significant distinction between the genuine user and imposters (i.e., high intervariance).

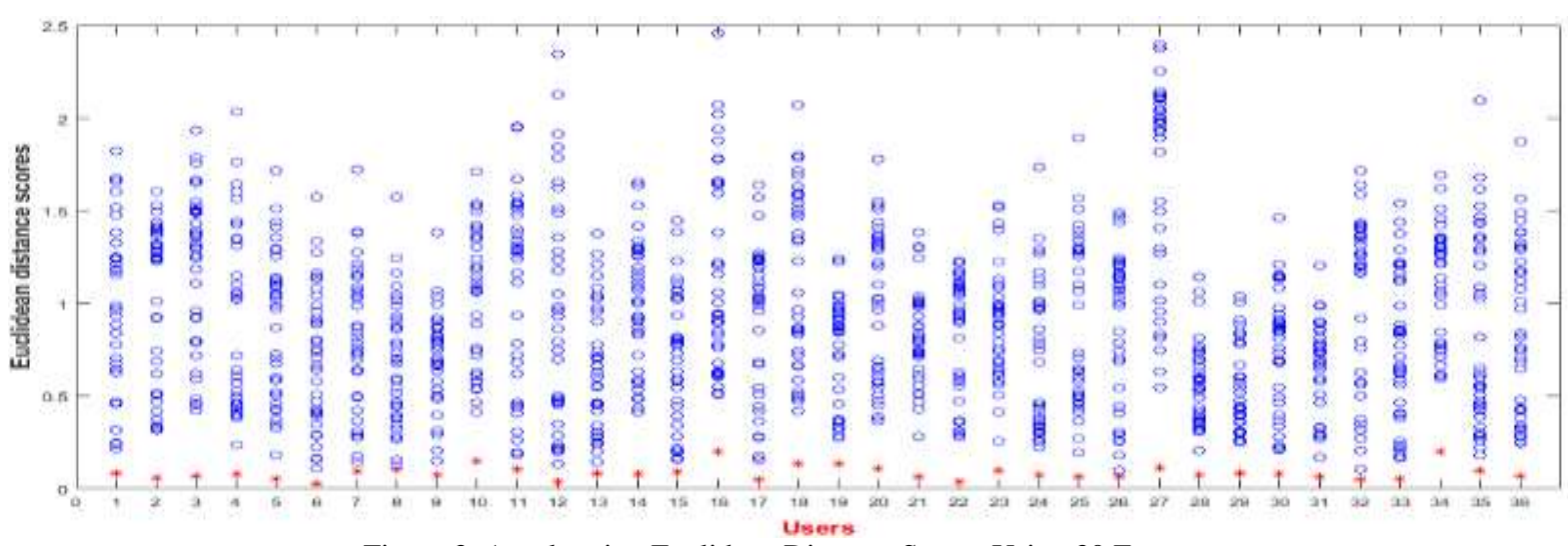

Figure 3. Acceleration Euclidean Distance Scores Using 30 Features

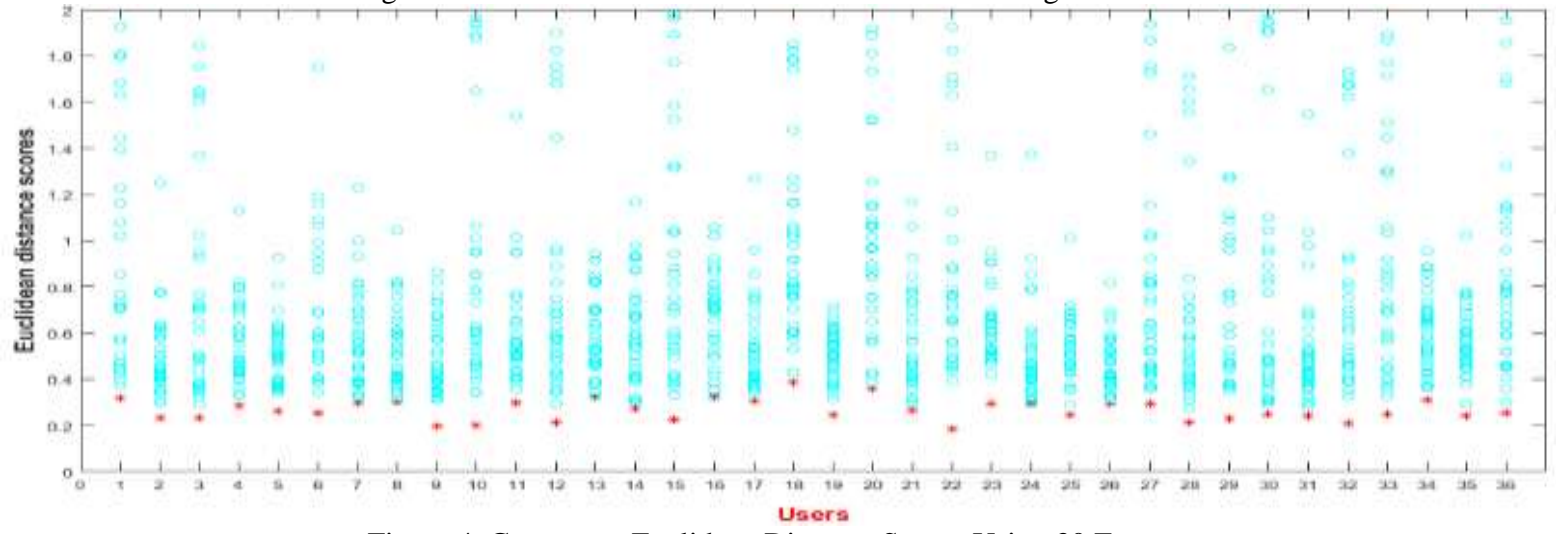

Figure 4. Gyroscope Euclidean Distance Scores Using 30 Features

\section{Proposed Architecture to support Smartwatch-based Activity Recognition}

A high-level architecture of the proposed system is presented in Figure 5. The prior art has established that managing the complex and varying signals of real-life use is a significant barrier. In order to overcome this, a context aware approach will be used in order to predict the user's activity at a specific point of time. This can be achieved by obtaining information from other smartwatch sensors (e.g., GPS) and using the information to create a multiclassifier approach that is trained to specific activities. This should result in a reduction in the variability in the feature set and provide better classification performance.

Unlike most of the prior studies that utilized information from a single sensor only (i.e., accelerometer or gyroscope), the proposed system aims to collect the movement data of both sensors as well as GPS information.

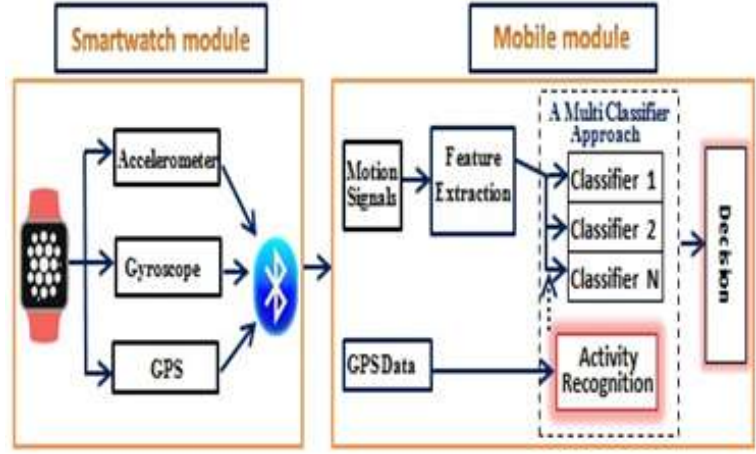

Figure 5. Proposed Architecture for Motion-based Activity Recognition

It is possible that the fusion of acceleration and gyroscope data would offer a greater level of accuracy than either sensor alone. Thereafter, feature selection needs to be sophisticated enough before the classification phase takes place. This can be achieved by selecting the features that are more resistant to changes of the user's behaviour. Finally, a set of classification methods will be evaluated to create a model for each individual activity 


\section{Conclusion and Future Work}

In the experimental study, movement data was collected from 36 subjects and the feature set analysed to determine its uniqueness. The data collection process was more realistic than previous studies [20], as each subject was asked to walk a predefined route that included flat ground/ multiple turns and opening doors. The results of this paper show that smartwatch motion sensors (i.e., accelerometer and gyroscope) can be effectively used in a Transparent Authentication System and future work needs to focus upon developing appropriate classification strategies to maximise performance. The study also shows some good results using the more realistic Cross-day scenario by utilizing small feature subsets. Unlike most of the previous smartphone based activity recognition systems, the proposed feature selection method utilized a dynamic feature vector for each user in order to have a trade-off between FAR and FRR. This feature reduction will help to decrease the computation burden of creating the test template on smartphones and/or smartwatches. However, more experimental work is required to evaluate whether the selected features of this study are the most effective feature sets. This can be carried out by using advanced techniques (e.g., decision-tree based classifiers and neural networks).

Unlike most existing motion-based authentication studies implemented within a controlled environment (i.e., all participants were asked to perform specific activities in an indoor environment), future work will also aim to design a methodology in order to collect real life data (i.e., users would wear a smartwatch during their day-to-day activities). By collecting unconstrained data a richer user profile can be generated. This could be extended to include interacting and typing on the smartphone touch screen and collecting different walking paces. As the nature of the real life signals is likely to be noisy, data from other smartwatch sensors (e.g., GPS) could be used in order to develop a context-aware approach (which will be useful to predict the user's activity).

\section{Acknowledgment}

The authors would like to thank the test subjects who participated in this study. I would also like to express my sincere gratitude to the University of Kufa for their financial support of this research.

\section{References}

[1]THE RADICATI GROUP, INC., (2015). Mobile Statistics Report, 2015-2019. [online] LONDON, UK, pp.2-3. Available at: http://www.radicati.com/wp/wpcontent/uplo ads/2015/02/Mobile-Statistics-Report-20152019-Executive-Summary.pdf [Accessed 30 Jan. 2017].
[2] Lifestylegroup, "Data stored on a phone more precious than the phone itself," 2011 .[Online]. Available: http://www.lifestylegro up.co.uk/content/Data-stored-ona-phone-more-precious-than-the-phone-itself.html.

[Accessed: 27-Jan-2017].

[3] N. L. Clarke and S. M. Furnell, "Advanced user authentication for mobile devices", Computer and Security, vol. 26, no. 2, pp. 109-119, 2007.

[4] M. Hamblen, "Mobile phone security no-brainer: Use a device passcode", 2013. [Online]. Available: http://www. computerworld.com/article/2497183/mobile-security/ mobile-phone-security-no-brainer--use-a-device-passcode. html. [Accessed:25-Jan-2017].

[5] N. Clarke, Transparent user authentication: biometrics, RFID and behavioural profiling. Springer London, ISBN: 978-0-85729-805-8, 2011.

[6] H. Saevanee, N. L. Clarke, and S. M. Furnell, "Multimodal Behavioural Biometric Authentication for Mobile Devices", in IFIP Advances in Information and Communication Technology, vol. 376 AICT, 2012, pp. 465-474.

[7] PWC, (2016). The Wearable Future. [online] United States, pp.4-6. Available at:

https://www.pwc.com/us/en/technology /publications/ assets/pwc-wearable-tech-design-oct-8th.pdf [Accessed 30 Jan. 2017].

[8] L. Rong, D. Zhiguo, Z. Jianzhong, and L. Ming, "Identification of Individual Walking Patterns Using Gait Acceleration", in 2007 1st International Conference on Bioinformatics and Biomedical Engineering, 2007, pp. 543-546.

[9] D. Gafurov, E. Snekkenes, and P. Bours, "Spoof Attacks on Gait Authentication System", IEEE Transactions on. Information. Forensics Security., vol. 2, no. 3, pp. 491-502, Sep. 2007.

[10] M. O. Derawi, P. Bours, and K. Holien, "Improved Cycle Detection for Accelerometer Based Gait Authentication", in 2010 Sixth International Conference on Intelligent Information Hiding and Multimedia Signal Processing, 2010, pp. 312-317.

[11] M. O. Derawi, C. Nickel, P. Bours, and C. Busch, "Unobtrusive User-Authentication on Mobile Phones Using Biometric Gait Recognition", in 2010 Sixth International Conference on Intelligent Information Hiding and Multimedia Signal Processing, 2010, pp. 306-311.

[12] J. R. Kwapisz, G. M. Weiss, and S. A. Moore, "Cell phone-based biometric identification", in 2010 Fourth IEEE International Conference on Biometrics: Theory, Applications and Systems (BTAS), 2010, pp. 1-7.

[13] C. Nickel, H. Brandt, and C. Busch, "Benchmarking the performance of SVMs and HMMs for accelerometerbased biometric gait recognition", in 2011 IEEE International Symposium on Signal Processing and Information Technology (ISSPIT), 2011, pp. 281-286. 
[14] C. Nickel, M. O. Derawi, P. Bours, and C. Busch, "Scenario test of accelerometer-based biometric gait recognition", in 2011 Third International Workshop on Security and Communication Networks (IWSCN), 2011, pp. 15-21.

[15]C. Nickel and C. Busch, "Classifying accelerometer data via Hidden Markov Models to authenticate people by the way they walk", in 2011 Carnahan Conference on Security Technology, 2011, vol. 28, no. 10, pp. 1-6.

[16] M. Muaaz and R. Mayrhofer, "An Analysis of Different Approaches to Gait Recognition Using Cell Phone Based Accelerometers", in Proceedings of International Conference on Advances in Mobile Computing \& Multimedia - MoMM '13, 2013, pp. 293300.

[17] T. Hoang, T. Nguyen, C. Luong, S. Do, and D. Choi, "Adaptive Cross-Device Gait Recognition Using a Mobile Accelerometer", Jornal of Information. Processing System., vol. 9, no. 2, pp. 333-348, Jun. 2013.

[18] A. H. Johnston and G. M. Weiss, "Smartwatch-based biometric gait recognition," in 2015 IEEE 7th International Conference on Biometrics Theory, Applications and Systems (BTAS), 2015, pp. 1-6.

[19] G. M. Weiss, J. L. Timko, C. M. Gallagher, K. Yoneda, and A. J. Schreiber, "Smartwatch-based activity recognition: A machine learning approach", in 2016 IEEEEMBS International Conference on Biomedical and Health Informatics (BHI), 2016, pp. 426-429.

[20] N. Al-Naffakh, N. Clarke, P. Dowland, and F. Li ACTIVITY RECOGNITION USING WEARABLE COMPUTING. Barcelona, Spain: ICITST-2016, pp.189195. Available at: http://icitst.org/ICITST-2016Proceedings /ICITST-2016-Proceedings.pdf. 